\title{
Model Checking Recursive Programs with Exact Predicate Abstraction
}

\author{
Arie Gurfinkel ${ }^{1}, \mathrm{Ou} \mathrm{Wei}^{2}$, and Marsha Chechik ${ }^{2}$ \\ ${ }^{1}$ Software Engineering Institute, Carnegie Mellon University \\ ${ }^{2}$ Department of Computer Science, University of Toronto
}

\begin{abstract}
We propose an approach for analyzing non-termination and reachability properties of recursive programs using a combination of over- and underapproximating abstractions. First, we define a new concrete program semantics, mixed, that combines both natural and operational semantics, and use it to design an on-the-fly symbolic algorithm. Second, we combine this algorithm with abstraction by following classical fixpoint abstraction techniques. This makes our approach parametrized by different approximating semantics of predicate abstraction and enables a uniform solution for over- and under-approximating semantics. The algorithm is implemented in YASM, and we show that it can establish non-termination of non-trivial C programs completely automatically.
\end{abstract}

\section{Introduction}

Automated predicate abstraction is one of the key techniques for extending finite-state model-checking to software. It combines automated construction of a finite abstract model with automated analysis by model-checking and iterative abstraction refinement. Traditionally, predicate abstraction is an over-approximation of a program and thus is biased towards establishing correctness of safety properties. To exploit the bug detection ability of model-checkers and to extend the scope of abstract model-checkers to richer properties, recent research has proposed abstract analysis that combines both over- and under-approximations $[9,15,25,26,4,18,17]$. Although such a combination, which we call exact-approximation, has been shown to be effective in practice [17, 19], until now this line of research has focused exclusively on analyzing non-recursive programs. In this paper, we propose a novel approach to extend such over- and underapproximating analyses to recursive programs. Our approach has been implemented in a software model-checker YASM. We illustrate it on non-termination and reachability analysis of several C programs, including the benchmarks from BEBOP [6], VERA [1], and MOPED [14, 8], the Ack program from [10] and a buggy version of Quicksort from [14]. To our knowledge, this is the first time that non-termination of such C programs was established completely automatically.

As a motivation, we review an over-approximation-based approach for model-checking of non-recursive programs and its limitations. Assume we want to check whether the ERROR label is reachable in the $\mathrm{C}$ program $\mathrm{EX}_{0}$ shown in Figure 1(a). This safety property is expressed in CTL as $\varphi: A G(p c \neq \mathrm{ERROR})$. An over-approximating abstraction $\alpha\left(\mathrm{EX}_{0}\right)$ of $\mathrm{EX}_{0}$ using the predicate $p: x>0$ is shown in Figure 1(b), where ' $\star$ ' is interpreted as a non-deterministic choice. $\alpha\left(\mathrm{EX}_{0}\right)$ is a finite boolean model which over-approximates the original program: it contains all feasible and some infeasible (or spurious) executions. For example, $\alpha\left(\mathrm{EX}_{0}\right)$ has an execution which gets 

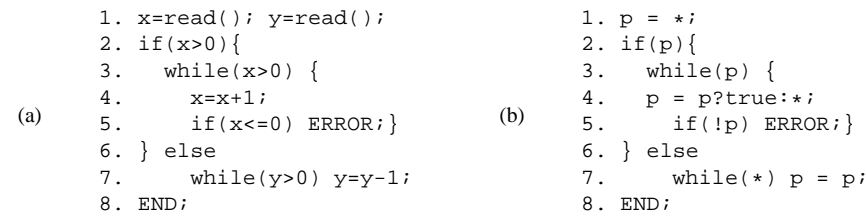

Fig. 1. (a) A program $\mathrm{EX}_{0}$, and (b) its over-approximation $\alpha\left(\mathrm{EX}_{0}\right)$ using predicate $p: x>0$.

stuck in the while $(*)$ loop on line 7 , but $\mathrm{EX}_{0}$ does not have the corresponding execution. Thus, if a universal temporal property, i.e., in the one expressed in ACTL, holds in $\alpha\left(\mathrm{EX}_{0}\right)$, it also holds in $\mathrm{EX}_{0}$. For example, our property $\varphi$ is satisfied by $\alpha\left(\mathrm{EX}_{0}\right)$, which means $\mathrm{ERROR}$ is unreachable in $\mathrm{EX}_{0}$. However, when a property is falsified by $\alpha\left(\mathrm{EX}_{0}\right)$, the result cannot be trusted since it may be caused by a spurious behavior. For example, consider checking whether $\mathrm{EX}_{0}$ always terminates, i.e., whether it satisfies $\psi: A F(p c=\mathrm{END}) . \psi$ is falsified on our abstraction, but this result cannot be trusted due to the infeasible non-terminating execution around the while (*) loop on line 7.

The falsification (or refutation) ability of predicate abstraction can be dramatically improved by using an under-approximating abstraction, where each abstract behavior is simulated by some concrete one. In this case, if a bug (or an execution) is present in the abstract model, it must exist in the concrete program. For example, the predicate $p$ must always be true in the while (p) loop at line 3 (assuming int is interpreted as mathematical integers). Thus, an under-approximation based on predicate $p$ is sufficient to establish that $\mathrm{EX}_{0}$ is non-terminating.

There has been a considerable amount of research exploring abstract analysis based on a combination of over- and under-approximating abstractions, e.g., [9, 15, 25, 26, 4, $18,17]$. Compared with an analysis based on over-approximation alone, there are two main differences:

1. Such a combination requires a non-boolean abstract model that can represent both over- and under-approximations at the same time. Examples of such models are Modal Transition Systems [21] (equivalently, 3-valued Kripke structures [9]) and Mixed Transition Systems [13] (equivalently, 4-valued Kripke structures [18]). These models use two types of transitions: may for over-approximation, and must for under-approximation.

2. It requires new model-checking algorithms for these models, such that a formula is evaluated to either true or false, which are trusted, or to unknown, which indicates that the abstraction is not precise enough for a conclusive analysis.

Although both theoretical and practical combinations of exact-approximation with automated CounterExample Guided Abstraction Refinement have been explored, they are all limited to analyzing non-recursive programs.

One way to extend such analysis to recursive programs is to continue to mirror the traditional approach, i.e., (a) extend push-down systems to support combined overand under-approximations, and (b) develop analysis algorithms for this new modeling formalism. While this approach seems natural, we are not aware of any existing work along this line.

In this paper, we propose an alternative solution to this problem. Our approach does not require the development of new specialized types of push-down systems, nor new specialized analysis algorithms. The key to our approach is to separate the analy- 
sis of recursive programs from abstraction of the data domain. We accomplish this by introducing a new concrete program semantics, which we call mixed, and using it to derive efficient symbolic algorithms for the analysis of non-termination and reachability properties of finite recursive programs. These algorithms share many insights with techniques in related work $[8,6,1]$, i.e., they are functional [24] in terms of interprocedural analysis, and apply only to stack-independent properties. The novelty of our approach is the formalization of the algorithms as equational systems, and the parametrization of the algorithms by data abstractions. This makes it possible to share the same analysis algorithms for over-, under-, and exact-approximations! In particular, we demonstrate that in combination with exact-approximation [17], our abstract analysis supports both verification and refutation.

The rest of this paper is organized as follows. We present preliminaries and fix our notation in Sec. 2. We present a simple programming language PL and its natural, and operational semantics in Sec. 3. In Sec. 4, we introduce mixed semantics and derive symbolic on-the-fly algorithms for analyzing recursive programs with finite data domain for reachability and non-termination. In Sec. 5, we parametrize the algorithms of Sec. 4 by abstraction for handling programs with infinite data domain. Experiments are reported in Sec. 6, and we conclude in Sec. 7. Additional illustrations are given in the Appendix.

\section{Preliminaries}

Valuation and Relations. A valuation $\sigma$ on a set of typed variables $V$ is a function that maps each variable $x$ in $V$ to a value $\sigma(x)$ in its domain. We assume that valuations extend to expressions in the obvious way. The domain of $\sigma$ is called a valuation type and is denoted by $\tau(\sigma)$. For example, if $\sigma=\{x \mapsto 5, y \mapsto 10\}$ then $\tau(\sigma)=\{x, y\}$. The projection of $\sigma$ on a subset $U \subseteq V$ is denoted by $\left.\sigma\right|_{U}$.

The set of all valuations over $V$ is denoted by $\Sigma_{V} \triangleq\{\sigma \mid \tau(\sigma)=V\}$. Note that $\Sigma_{\emptyset}$ is well-defined and consists of the unique empty valuation. A relation $r$ on two sets of variables $U$ and $V$ is a subset of $\Sigma_{U} \times \Sigma_{V}$. The relational type of $r$ is $U \rightarrow V$, denoted by $\tau(r)$. For example, the type of $x^{\prime}=y$ is from $y$ to $x$, that is, $\tau\left(x^{\prime}=y\right)=\{y\} \rightarrow\{x\}$. In this paper, we use several simple relations: true is the true relation, $i d$ is the identity relation (e.g., $i d(x) \triangleq x^{\prime}=x$ ), decl is a relation for variable declaration, and kill - for variable elimination. Formally, they are defined as follows, with the format name ' $\triangleq$ ' expression ':' type:

$$
\begin{array}{lll}
\operatorname{true}(U \rightarrow V) & \triangleq \Sigma_{U} \times \Sigma_{V}: U \rightarrow V & \operatorname{decl}(V) \triangleq \operatorname{true}(\emptyset \rightarrow V): \emptyset \rightarrow V \\
\operatorname{kill}(V) & \triangleq \operatorname{true}(V \rightarrow \emptyset): V \rightarrow \emptyset \quad \operatorname{id}(V) \triangleq\left\{\left(\sigma, \sigma^{\prime}\right) \in \Sigma_{V} \times \Sigma_{V} \mid \sigma=\sigma^{\prime}\right\}: V \rightarrow V
\end{array}
$$

Operations on relations are defined in Table 1 , where $\vee, \circ$ and $\times$ are asynchronous, sequential and parallel composition, respectively, assume is a restriction of identity relation to a set $Q$ of valuations, [.] is variable introduction, and $(\cdot \rightarrow \cdot)$ is scope extension. Note that $\times$ combines the outputs of two relations, and [.] extends the source of a relation with new variables. Together these operators allow constructing complex relations from simple ones. For example, $[\{x, y\}]\left(x^{\prime}=y\right) \times[\{x, y\}]\left(y^{\prime}=x\right)$ is the relation $\left(x^{\prime}=y\right) \wedge\left(y^{\prime}=x\right)$ with the type $\{x, y\} \rightarrow\{x, y\}$. Directly composing $x^{\prime}=y$ and $y^{\prime}=x$ without variable introduction, i.e., $\left(x^{\prime}=y\right) \times\left(y^{\prime}=x\right)$, is invalid because $\tau\left(x^{\prime}=y\right)=\{y\} \rightarrow\{x\}$ and $\tau\left(y^{\prime}=x\right)=\{x\} \rightarrow\{y\}$ have different source types. 


\begin{tabular}{|c|c|c|c|}
\hline Operation & Assumption & Definition & Type \\
\hline$r_{1} \vee r_{2}$ & $\tau\left(r_{1}\right)=\tau\left(r_{2}\right)$ & $\lambda a, a^{\prime} \cdot r_{1}\left(a, a^{\prime}\right) \vee r_{2}\left(a, a^{\prime}\right)$ & $\tau\left(r_{1}\right)$ \\
\hline$r_{1} \circ r_{2}$ & $\tau\left(r_{1}\right)=U \rightarrow V$ & $\lambda a, a^{\prime} \cdot \vee_{a^{\prime \prime}}\left(r_{1}\left(a, a^{\prime \prime}\right) \wedge r_{2}\left(a^{\prime \prime}, a^{\prime}\right)\right)$ & $U \rightarrow W$ \\
\hline & $\wedge \tau\left(r_{2}\right)=V \rightarrow W$ & & \\
& $\tau\left(r_{1}\right)=U \rightarrow V_{1}$ & $\lambda a, a^{\prime} \cdot r_{1}\left(a,\left.a^{\prime}\right|_{V_{1}}\right) \wedge r_{2}\left(a,\left.a^{\prime}\right|_{V_{2}}\right)$ & $U \rightarrow\left(V_{1} \cup V_{2}\right)$ \\
$r_{1} \times r_{2}$ & $\wedge \tau\left(r_{2}\right)=U \rightarrow V_{2}$ & & \\
\hline assume $(Q)$ & $\wedge V_{1} \cap V_{2}=\emptyset$ & $\lambda a, a^{\prime} \cdot Q(a) \wedge i d(\tau(Q))\left(a, a^{\prime}\right)$ & $\tau(Q) \rightarrow \tau(Q)$ \\
\hline$[W] r$ & & $\lambda a, a^{\prime} \cdot r\left(\left.a\right|_{U}, a^{\prime}\right)$ & $(U \cup W) \rightarrow V$ \\
\hline$(W \rightarrow Z) r$ & $\tau(r)=U \rightarrow V \wedge U \subseteq W \wedge(Z \backslash V) \subseteq W$ & $([W] r) \times([W](i d(Z \backslash V)))$ & $W \rightarrow Z$ \\
\hline
\end{tabular}

Table 1. Relational operations.

Scope extension extends a relation by combining it with the identity on new variables. For example, $(\{x, y\} \rightarrow\{x, y\})\left(x^{\prime}=x+1\right)$ is $\left(x^{\prime}=x+1\right) \wedge\left(y^{\prime}=y\right)$. The assumptions for scope extension ensure that any new variable introduced in the destination of $r$ must also be available in the source. For example, the extension $(\{x, y\} \rightarrow$ $\{x, z\})\left(x^{\prime}=x+1\right)$ is not allowed since $z$ is not available in the source of the relation.

For a relation $r$ with a type $U \rightarrow V$, we define the pre-image of $Q \subseteq \Sigma_{V}$ w.r.t. $r$, $\operatorname{pre}[r]: \mathbf{2}^{\Sigma_{V}} \rightarrow \mathbf{2}^{\Sigma_{U}}$, as

$$
\operatorname{pre}[r](Q) \triangleq \lambda a \cdot \vee_{a^{\prime}}\left(r\left(a, a^{\prime}\right) \wedge Q\left(a^{\prime}\right)\right)
$$

Reachability and Non-termination. A Kripke structure $K=\langle\mathcal{S}, \mathcal{R}\rangle$ is a transition system, where $\mathcal{S}$ is a set of states and $\mathcal{R} \subseteq \mathcal{S} \times \mathcal{S}$ is a transition relation.

Let $p$ be an atomic proposition, and $\mathcal{S}_{p} \triangleq\{s \in \mathcal{S} \mid s \models p\}$ be the set of states satisfying $p$. A reachability property ( $E F p$ in CTL) is true in a state $s$ if there exists a path from $s$ to a state in $\mathcal{S}_{p}$. A non-termination property ( $E G p$ in CTL) is true in a state $s$ if there exists an infinite path starting at $s$ and contained in $\mathcal{S}_{p}$.

The set $R S$ of all states satisfying $E F p$ is the least solution to equation reach, and the set $N T$ of all states satisfying $E G p$ is the greatest solution to equation non-term:

$$
R S=\mathcal{S}_{p} \cup \operatorname{pre}[\mathcal{R}](R S) \quad \text { (reach) } \quad N T=\operatorname{pre}\left[\mathcal{R} \cap \mathcal{S}_{p}\right](N T) \text { (non-term) }
$$

\section{Programming Language and Semantics}

We use a simple imperative programming language PL which allows non-determinism and recursive function calls. We assume that (a) functions have a set of call-by-value formal parameters and a set of return variables; (b) each variable has a unique name and explicit scope; (c) there are no global variables (they can be simulated by local variables); and (d) a type expression is associated with each statement and explicitly defines the pre- and post-variables of the statement.

Syntax. Let var denote variables, func function identifiers, $e$ expressions, and $T$ valuation types. The syntax of PL is defined as follows:

$$
\begin{aligned}
\text { Atomic }::= & \text { skip } \mid \text { var }^{+}:=e^{+}|\operatorname{assume}(e)| \operatorname{var} \text { var }^{+} \mid \text {kill } \text { var }^{+} \mid(T \rightarrow \text { T)Atomic } \\
\text { Stmt }::= & \text { Atomic } \mid \text { Stmt } ; \text { Stmt } \mid \text { Stmt }|| \text { Stmt } \mid \text { if }(e) \text { then Stmt else Stmt } \\
& \mid \text { while }(e) \text { Stmt } \mid \text { var }^{+}:=\text {func }\left(\text { var }^{+}\right) \mid(T \rightarrow T) \text { Stmt } \\
\text { Fdef }::= & \text { func }\left(\text { var }^{+}\right): \text {var }^{+} \text {Stmt } \\
\text { Prog }::= & \text { Fdef }
\end{aligned}
$$

We use bold lower case letters to represent vectors, e.g., a statement $\mathbf{x}:=\mathbf{e}$ means an assignment $x_{1}, \cdots, x_{n}:=e_{1}, \cdots, e_{n}$. For a function $f$ with declaration $f\left(p_{1}, \cdots, p_{n}\right)$ : 

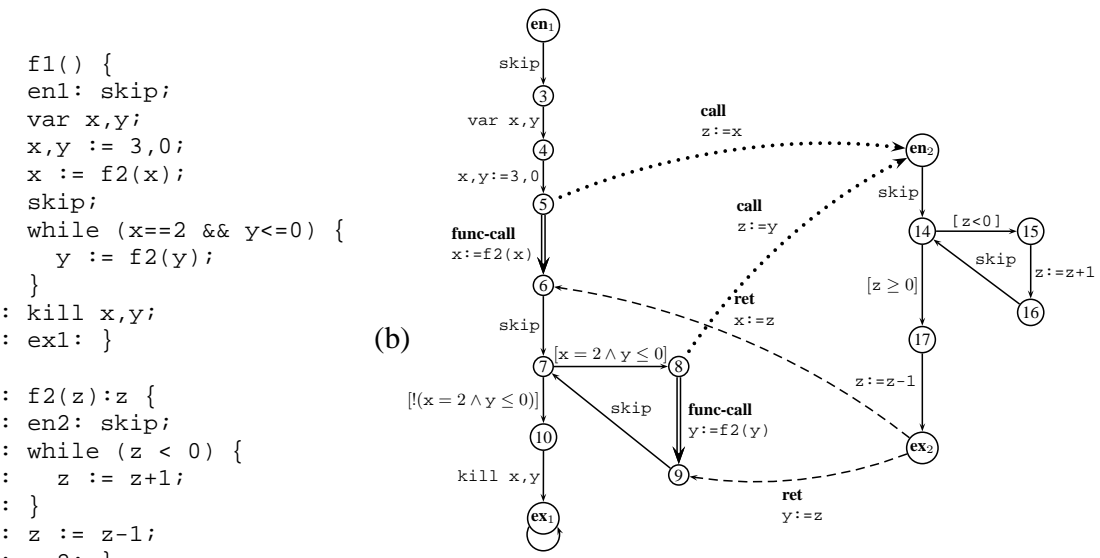

Fig. 2. (a) A program $\mathrm{EX}_{1}$ and (b) its ICFG.

$r_{1}, \cdots, r_{k}, \mathbf{p}_{f}$ and $\mathbf{r}_{f}$ to denote the formal parameters and the return variables of $f$, respectively. $\operatorname{var}(e)$ denotes the variables of $e$, and we assume that each program has a "main" function $f_{1}$, not called by other functions.

Base Semantics. Let $\Sigma$ denote the set of all valuations in a PL program. With each atomic statement $S$, we associate base semantics that interprets the statement as a relation $\llbracket S \rrbracket \subseteq \Sigma \times \Sigma$ on valuations of program variables:

$$
\begin{gathered}
\llbracket \mathbf{s k i p} \rrbracket \triangleq i d(\emptyset) \llbracket \operatorname{var} \mathbf{x} \rrbracket \triangleq \operatorname{decl}[\mathbf{x}] \quad \llbracket \mathbf{k i l l} \mathbf{x} \rrbracket \triangleq \operatorname{kill}[\mathbf{x}] \llbracket(U \rightarrow V)(S) \rrbracket \triangleq(U \rightarrow V) \llbracket S \rrbracket \\
\llbracket \mathbf{x}:=\mathbf{e} \rrbracket \triangleq\left\{\left(\sigma, \sigma^{\prime}\right) \mid \tau(\sigma)=\operatorname{var}(\mathbf{e}) \wedge \sigma^{\prime}=\left[\mathbf{x}_{i} \mapsto \sigma\left(\mathbf{e}_{i}\right)\right]\right\} \\
\llbracket \mathbf{a s s u m e}(e) \rrbracket \triangleq\left\{\left(\sigma, \sigma^{\prime}\right) \mid\left(\sigma, \sigma^{\prime}\right) \in i d(\operatorname{var}(e)) \wedge \sigma \models e\right\}
\end{gathered}
$$

Note that for the type cast statement $(U \rightarrow V) S$, we only consider those cases where the assumptions for the scope extension are satisfied.

Interprocedural Control Flow Graph. A PL program is represented by an Interprocedural Control Flow Graph (ICFG) [24]. An ICFG is a labeled graph $G=\langle L o c, E d g e, \pi\rangle$, where $L o c$ is a finite set of locations, $E d g e \subseteq L o c \times L o c$ is a set of edges, and $\pi$ labels each edge with a program statement. For example, the ICFG for the program $\mathrm{EX}_{1}$ (see Fig. 2(a)) is shown in Fig. 2(b). In ICFGs, (a) each function has a unique entry (en) and exit (ex); (b) there is a self-loop at ex of $f_{1}$ to ensure existence of an infinite execution; (c) each function call (func-call) is: a call edge, where the values of actual parameters of the callee function are assigned to the formal parameters, a function body, and a ret edge, where the return values are assigned to the variables of the caller.

We assume that call and ret edges are uniquely determined by each other. For a call edge $(k, \mathbf{e n})$ and the corresponding ret edge $(\mathbf{e x}, l), k$ is the call location, $\operatorname{call}(l) \triangleq k$, and $l$ is the return location, $\operatorname{ret}(k) \triangleq l$.

Operational Semantics of a program $P=\langle L o c, E d g e, \pi\rangle$ is a transition system $K=\langle\mathcal{S}, \mathcal{R}\rangle$. Each state in $\mathcal{S}$ is a stack of activation records where each record is of the form $\langle p c, \sigma\rangle$, where $p c \in L o c$ is a program counter, corresponding to a particular control location in $P$, and $\sigma \in \Sigma_{V(p c)}$ is the valuation for variables in the scope of $p c$ (denoted by $V(p c)$ ). For a state $s=\left(k, \sigma_{k}\right) . \Gamma,\left(k, \sigma_{k}\right)$ is the top element of $s, \operatorname{top}(s)$. For a pair of states $s=\left(k, \sigma_{k}\right) \cdot \Gamma_{s}$ and $t=\left(l, \sigma_{l}\right) \cdot \Gamma_{t}$, the transition relation $\mathcal{R}$ is defined as $\mathcal{R}(s, t) \triangleq\langle k, l\rangle \in E d g e \wedge r_{\langle k, l\rangle}(s, t)$, where $r_{\langle k, l\rangle}$ is a deterministic (but not neces- 


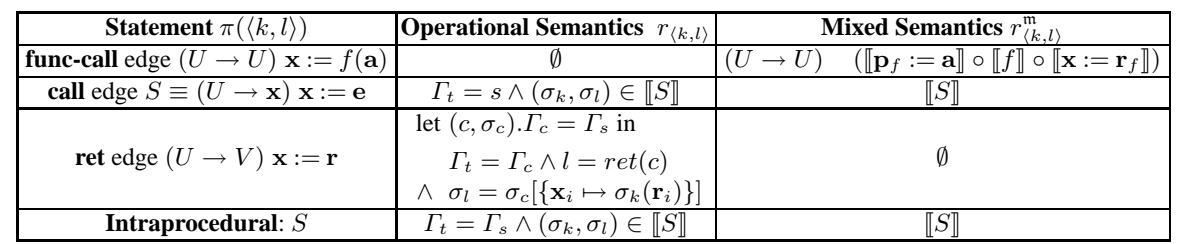

Table 2. The rules of operational and mixed semanics. $U$ is the set of local variables in the scope of the function call; $\llbracket f \rrbracket$ is natural semantics, $\mathbf{p}_{f}$ are the formals, and $\mathbf{r}_{f}$ are the returns of $f$.

sarily total) relation on $\mathcal{S}$ at the edge $\langle k, l\rangle$, as defined in the 2 nd column of Table 2 . An intraprocedural statement only modifies the top activation record, and a statement on a call or a ret edge pushes a new record or pops one, respectively. The transition relations on func-call edges are empty, i.e., these edges are removed.

Natural Semantics [22] (a.k.a. big-step) of a block of code $S$ is a relation $\llbracket S \rrbracket \subseteq \Sigma \times \Sigma$ between the input and output of $S$ : i.e., $\left(\sigma, \sigma^{\prime}\right) \in \llbracket S \rrbracket$ iff the execution of $S$ on $\sigma$ terminates and results in $\sigma^{\prime}$. Natural semantics of a program $P \equiv f_{1}, \cdots, f_{n}$ is a set of relations, one per function, i.e., $\llbracket P \rrbracket=\left\langle\llbracket f_{1} \rrbracket, \cdots, \llbracket f_{n} \rrbracket\right\rangle$.

The semantic rules for PL are defined compositionally on the syntax using the function $\llbracket \cdot \rrbracket_{\rho}$, where $\rho$ is an environment mapping free fixpoint variables (used for loops and functions) to relations with an appropriate type. Natural semantics for atomic statements is the same as base semantics; the other cases are:

$$
\begin{array}{cl}
\llbracket S_{1} ; S_{2} \rrbracket_{\rho} \triangleq \llbracket S_{1} \rrbracket_{\rho} \circ \llbracket S_{2} \rrbracket_{\rho} & \llbracket \mu X \cdot \mathcal{S}(X) \rrbracket_{\rho} \triangleq \operatorname{lfp}\left(\lambda Z \cdot \llbracket \mathcal{S}(X) \rrbracket_{\rho\{X \mapsto Z\}}\right) \\
\llbracket S_{1} \| S_{2} \rrbracket_{\rho} \triangleq \llbracket S_{1} \rrbracket_{\rho} \vee \llbracket S_{2} \rrbracket_{\rho} & \llbracket \mathbf{x}:=f(\mathbf{a}) \rrbracket_{\rho} \triangleq \llbracket \mathbf{p}_{f}:=\mathbf{a} ; X_{f} ; \mathbf{x}:=\mathbf{r}_{f} \rrbracket_{\rho} \\
\llbracket X \rrbracket_{\rho} \triangleq \rho(X) & \llbracket \text { while }(e) S \rrbracket_{\rho} \triangleq \llbracket \mu X_{w} \cdot \mathbf{i f}(e) \text { then }\left(S ; X_{w}\right) \rrbracket_{\rho} \\
\llbracket \mathbf{i f}(e) \text { then } S_{1} \text { else } S_{2} \rrbracket_{\rho} \triangleq \llbracket\left(\text { assume }(e) ; S_{1}\right) \|\left(\text { assume }(\neg e) ; S_{2}\right) \rrbracket_{\rho}
\end{array}
$$

where Ifp denotes for least fixpoint, $\tau\left(\rho\left(X_{f}\right)\right)=\mathbf{p}_{f} \rightarrow \mathbf{r}_{f}$ and $\tau\left(\rho\left(X_{w}\right)\right)=\tau\left(\llbracket S \rrbracket_{\rho}\right)$. A program $P \equiv f_{1}, \cdots, f_{n}$ induces the system of equations

$$
\rho\left(X_{f_{i}}\right)=\llbracket S_{f_{i}} \rrbracket_{\rho} \quad(1 \leq i \leq n)
$$

Natural semantics of $P$ is the least fixpoint solution to this system, e.g., for the program $\mathrm{EX}_{1}$, natural semantics of $f_{2}$ is $\left(z>0 \wedge z^{\prime}=z-1\right) \vee\left(z \leq 0 \wedge z^{\prime}=-1\right)$.

Theorem 1. Let $P \equiv f_{1}, \cdots, f_{n}$ be a program and $K=\langle\mathcal{S}, \mathcal{R}\rangle$ be its operational semantics. A pair of activation records $\left(\left\langle k, \sigma_{k}\right\rangle,\left\langle l, \sigma_{l}\right\rangle\right)$ is in $\llbracket f_{i} \rrbracket$ iff there exists a path $s_{0}, \cdots, s_{m}$ in $K$ such that $s_{0}=\left\langle k, \sigma_{k}\right\rangle . \Gamma_{0}$ and $s_{m}=\left\langle l, \sigma_{l}\right\rangle . \Gamma_{m}$, such that $\Gamma_{0}=\Gamma_{m}, k$ and $l$ are en and $\mathrm{ex}$ of $f_{i}$, respectively, and for all other $s_{j}=\left\langle p, \sigma_{p}\right\rangle \cdot \Gamma_{j}$ either $\Gamma_{j} \neq \Gamma_{0}$ or $p$ is not ex of $f_{i}$.

\section{Reachability and Non-Termination Analysis}

We now turn our attention to checking reachability and non-termination of recursive programs. Reachability can be reduced to finding the least fixpoint solution to the equation reach w.r.t. a transition system of operational semantics of a program (see Sec. 2). Similarly, non-termination corresponds to finding the greatest solution to the equation 
non-term. However, since operational semantics explicitly exposes a potentially unbounded call stack at each state, these equations must be solved over an infinite transition system (even when all program variables range over finite domains). Thus, the exact fixpoint solution may not be computable.

However, many program properties depend only on the top of the call stack: i.e., they are stack-independent. Analysis of such properties can be done using stack-free operational semantics in which everything except for the top activation record is abstracted away. In this section, we apply this idea to the analysis of $E F p$ (reachability) and $E G \quad p$ (non-termination) properties, where $p$ is a proposition that depends only on the top activation record. Without loss of generality, we further assume that $p$ only depends on program locations, i.e., it is of the form $p c=x$.

\subsection{Mixed Semantics}

We start by defining a stack-free operational semantics, called mixed semantics, for PL programs which removes the call stack but preserves reachability and non-termination properties w.r.t. operational semantics of Sec. 3 .

Intuitively, mixed semantics is a combination of operational and natural semantics, in which a program is executed as follows: an atomic statement is executed as usual; a function call $\mathrm{x}:=\mathrm{foO}(\mathrm{y})$ is executed as a non-deterministic choice between (a) executing foo, i.e., updating the top activation record according to natural semantics of foo, and (b) entering the body of foo, and forgetting all but the top activation record. Upon reaching the end of the main function, the execution enters a self-loop indicating the end of the program, and blocks at all other exit locations since it does not remember the origin of the call. For example, consider mixed execution of the program $\mathrm{EX}_{1}$ starting from line 5 with $x=3$ and $y=0$. At this point, the execution can either (a) move to line 6 and decrease $x$ by one according to natural semantics of $f_{2}$, or (b) move to en2 (line 13), assign $z$ to 3 , and forget about $x$ and $y$. Within $f_{2}$, the execution continues until it blocks at ex2 (line 18) with $z=2$.

Formally, mixed semantics of a program $P=\langle L o c, E d g e, \pi\rangle$ is a Kripke structure $K^{\mathfrak{m}}=\left\langle\mathcal{S}^{\mathfrak{m}}, \mathcal{R}^{\mathfrak{m}}\right\rangle$, where each state is a single activation record $\langle p c, \sigma\rangle$. For a pair of states $s=\left\langle k, \sigma_{k}\right\rangle$ and $t=\left\langle l, \sigma_{l}\right\rangle$, the transition relation is $\mathcal{R}^{\mathfrak{m}}(s, t) \triangleq$ $(\langle k, l\rangle \in E d g e) \wedge r_{\langle k, l\rangle}^{\mathfrak{m}}\left(\sigma_{k}, \sigma_{l}\right)$, where $r_{\langle k, l\rangle}^{\mathfrak{m}}$ is a relation on valuations, as defined in the 3rd column of Table 2. Note that $r_{e}^{\mathfrak{m}}$ for ret edges is empty, which is equivalent to removing those edges from the ICFG.

Mixed semantics preserves reachability and non-termination properties w.r.t. operational semantics. If an execution of a function $f$ reaches a state $s$ under the latter, then either $s$ is a location within $f$, or it is inside some other function that $f$ calls (directly or indirectly). The non-deterministic treatment of function calls in the former ensures that both of these cases are covered. Similarly, if there exists an infinite execution starting inside $f$, then either this execution lies within $f$, or $f$ calls a function that does not return the control back to $f$. Again, both cases are captured by mixed semantics.

Theorem 2. Let $K$ and $K^{\mathfrak{m}}$ be operational and mixed semantics of a given program, respectively, and p be a propositional formula on control locations. Then, $(K \models E F p) \Leftrightarrow$ $\left(K^{\mathfrak{m}} \models E F p\right)$ and $(K \models E G p) \Leftrightarrow\left(K^{\mathfrak{m}} \models E G p\right)$.

When all variables of a given program $P$ range over finite domains, mixed semantics of $P$ is a finite Kripke structure. Theorem 2 implies the following analysis algorithm: 
Step 1: compute natural semantics of $P$ by solving equation nat;

Step 2: construct the structure $K^{\mathfrak{m}}$ following the rules of mixed semantics;

Step 3: solve equations reach or non-term on $K^{\mathfrak{m}}$ for reachability or non-termination, respectively.

While sound and complete, this algorithm is not efficient, since it relies on the (potentially unnecessary) computation of "full" natural semantics of all functions (for Step 2 ) and the construction of "full" mixed semantics before the analysis of the property can even begin. As a trivial example, consider checking $E F(p c=5)$ on the program $\mathrm{EX}_{1}$. Since reachability of line 6 is irrelevant for this analysis, there is no need to construct the transition relation corresponding to func-call edge $\langle 5,6\rangle$ and thus no need to compute natural semantics of $f_{2}$. Following this observation, in the rest of this section, we show that the three steps of the above algorithm can be combined into an on-the-fly algorithm that only computes the necessary parts of mixed and natural semantics.

\subsection{On-the-fly Reachability}

Intuitively, the analysis of $E F p$ properties only needs a part of mixed semantics that is used for solving equation reach, and that, in turn, drives the computation of the necessary parts of natural semantics. To illustrate, consider checking $E F(p c=8)$ on $\mathrm{EX}_{1}$. Natural semantics of $f_{2}$ is $\llbracket f_{2} \rrbracket \equiv\left(z>0 \wedge z^{\prime}=z-1\right) \vee\left(z \leq 0 \wedge z^{\prime}=-1\right)$. After a few iterations, the reachability algorithm computes a pre-condition $Q \equiv x=2 \wedge y \leq 0$ for reaching line 8 from line 6 . To determine a pre-condition for $Q$ w.r.t. a function call $\mathrm{x}:=\mathrm{f} 2(\mathrm{x})$ at line 5 , it needs to compute $\operatorname{pre}\left[r_{\langle 5,6\rangle}^{\mathfrak{m}}\right](Q)=(x=3 \wedge y \leq 0)$, where $r_{\langle 5,6\rangle}^{\mathfrak{m}} \equiv\left(y^{\prime}=y\right) \wedge\left(\left(x>0 \wedge x^{\prime}=x-1\right) \vee\left(x \leq 0 \wedge x^{\prime}=-1\right)\right)$ is the instantiation of $\llbracket f_{2} \rrbracket$ to the call site. However, instead of using the "full" version of $\llbracket f_{2} \rrbracket$, it is sufficient to compute a pre-condition that assumes $Q$ as a post-condition, i.e., to restrict $r^{\mathfrak{m}}$ to $x^{\prime}=2$ (the relevant part of $Q$ ) yielding $\hat{r}^{\mathfrak{m}} \equiv y^{\prime}=y \wedge x=3 \wedge x^{\prime}=2 . \hat{r}^{\mathfrak{m}}$ is an instantiation of $z=3 \wedge z^{\prime}=2$ in the context of the call, obtained by (a) converting $Q$ to a postcondition of $f_{2}$ by taking its pre-image over the ret edge (which eliminates $y$ and renames $x$ to $z$ ), and (b) restricting $\llbracket f_{2} \rrbracket$ to this post-condition: $\llbracket f_{2} \rrbracket \circ(\operatorname{assume}(z=2)) \equiv z=3 \wedge z^{\prime}=2$.

We now formalize the above intuition. Recall that $V(k)$ stands for the set of variables in the scope of a location $k$. Let $l$ be the return location of a function call to $f_{i}, Q \subseteq$ $\Sigma_{V(l)}$ be a set of valuations at $l$, and the corresponding ret edge $\left\langle\mathbf{e x}_{i}, l\right\rangle$ be labeled with $\mathbf{x}:=\mathbf{r}_{f_{i}}$. Then, function $\operatorname{prop}\left(\left\langle\mathbf{e x}_{i}, l\right\rangle, Q\right) \triangleq \operatorname{pre}\left[\llbracket \mathbf{x}:=\mathbf{r}_{f_{i}} ;(\mathbf{x} \rightarrow V(l)) \operatorname{var}(V(l) \backslash \mathbf{x}) \rrbracket\right](Q)$ turns $Q$ into a post-condition of $f_{i}$. Here, the pre-image w.r.t. var undeclares (or removes) all variables that are not changed by the call, and the pre-image w.r.t. ret edge turns the post-condition on $\mathbf{x}$ into the one on $\mathbf{r}_{f_{i}}$.

Let $R S: L o c \rightarrow \mathbf{2}^{\Sigma}$ map each location $k$ to a subset of $\Sigma_{V(k)}$, and, as in Sec. 3, let $\rho$ be the semantics environment, mapping each fixpoint variable to a relation of an appropriate type. The on-the-fly algorithm for reachability analysis is the equation system reach-otf:

$$
\begin{aligned}
R S(k) & =\left\{\begin{array}{ll}
\Sigma_{V(k)} & \text { if } k \models p(k \in L o c) \\
R S(k) \cup \bigcup_{l \in \operatorname{succ}(k)} \operatorname{pre}\left[\hat{r}_{\langle k, l\rangle}^{\mathrm{m}}\right](R S(l)) & \text { otherwise }
\end{array} \quad\right. \text { (reach-otf) } \\
\rho\left(X_{f_{i}}\right) & =\llbracket S_{f_{i}} \rrbracket_{\rho} \circ \operatorname{assume}\left(\bigcup_{l \in \operatorname{succ}\left(\mathbf{e x}_{i}\right)} \operatorname{prop}\left(\left\langle\mathbf{e x}_{i}, l\right\rangle, R S(l)\right)\right) \quad(i \in[1 . . n])
\end{aligned}
$$


where succ are the successors of a node in the ICFG, $S_{f_{i}}$ is the body of $f_{i}$, and for $S \equiv \pi(\langle k, l\rangle), \hat{r}_{\langle k, l\rangle}^{\mathfrak{m}}$ is defined as:

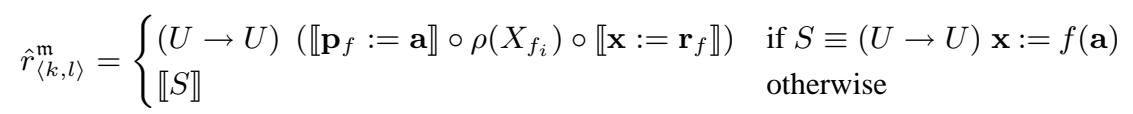

This system is a combination of nat and reach, where prop is used to propagate the reachability information to the computation of natural semantics. Since reachability and natural semantics are both least solutions to equations reach and nat, respectively, we need the least solution to the above equation as well.

The following theorem shows that the analysis based on equation system reach-otf is sound, and computes only the necessary part of natural semantics.

Theorem 3. Let $R S_{\downarrow}$ and $\rho_{\downarrow}$ be the least solutions to equation system reach-otf. Then,

1. $R S_{\downarrow}$ is the least solution to equation reach on $K^{\mathfrak{m}}$;

2. $\forall i \in[1 . . n] \cdot \rho_{\downarrow}\left(X_{f_{i}}\right) \subseteq \llbracket f_{i} \rrbracket$;

3. for any $\rho$, if $R S_{\downarrow}$ is the least solution to the $R S$ equations in reach-otf w.r.t. $\rho$, then $\forall i \in$ $[1 . . n] \cdot \rho_{\downarrow}\left(X_{f_{i}}\right) \subseteq \rho\left(X_{f_{i}}\right)$.

Part 1 of Theorem 3 shows that $R S_{\downarrow}$ is the solution for the reachability analysis; part 2 - that $\rho_{\downarrow}$ is sound w.r.t. natural semantics of $f_{i}$; and part 3 - that $\rho_{\downarrow}$ only contains the information necessary for the analysis.

Since we need the least solution for both $R S(k)$ and $\rho\left(X_{f_{i}}\right)$ equations, it can be obtained by any chaotic iteration [11] and thus is independent of the order of computation of $R S$ and $\rho$. Interestingly, the algorithm derived from reach-otf is a pre-image-based variant of the post-image-based reachability algorithm of BEBOP [6], and is similar to the formalization of backward analysis with wp described in [3].

\subsection{On-the-fly Non-Termination}

The derivation of the on-the-fly algorithm for the analysis of non-termination, nt-otf, proceeds similarly, and is a combination of systems nat and non-term:

$N T(k)= \begin{cases}\emptyset & \text { if } k \not \forall p(k \in L o c) \\ \bigcup_{l \in \operatorname{succ}(k)} \operatorname{pre}\left[\hat{r}_{\langle k, l\rangle}^{\mathfrak{m}}\right](N T(l)) & \text { otherwise }\end{cases}$

(nt-otf)

$\rho\left(X_{f_{i}}\right)=\llbracket S_{f_{i}} \rrbracket_{\rho} \circ$ assume $\left(\bigcup_{l \in \operatorname{succ}\left(\mathbf{e x}_{i}\right)} \operatorname{prop}\left(\left\langle\mathbf{e x}_{i}, l\right\rangle, N T(l)\right)\right) \quad(i \in[1 . . n])$

where $N T: L o c \rightarrow 2^{\Sigma}$ maps each location $k$ to a subset of $\Sigma_{V(k)}$, and succ, $S_{f_{i}}$ and $\hat{r}^{\mathfrak{m}}$ are the same as those in reach-otf. Since non-termination requires the greatest solution to non-term, and natural semantics - the least solution to nat, in nt-otf, we need the greatest solution to $N T(k)$, and the least solution to $\rho\left(X_{f_{i}}\right)$ equations, respectively.

The following theorem shows that the non-termination algorithm based on nt-otf is sound and computes only the necessary part of natural semantics.

Theorem 4. Let $N T_{\uparrow}$ and $\rho_{\downarrow}$ be the greatest solution for $N T$ and the least solution for $\rho$ in system nt-otf, respectively. Then,

1. $N T_{\uparrow}$ is the greatest solution to the equation non-term on $K^{\mathfrak{m}}$;

2. $\forall i \in[1 . . n] \cdot \rho_{\downarrow}\left(X_{f_{i}}\right) \subseteq \llbracket f_{i} \rrbracket$;

3. for any $\rho$, if $N T_{\uparrow}$ is the greatest solution to the $N T$ equations in $\mathrm{nt}$-otf w.r.t. $\rho$, then $\forall i \in$ $[1 . . n] \cdot \rho_{\downarrow}\left(X_{f_{i}}\right) \subseteq \rho\left(X_{f_{i}}\right)$. 
As in Theorem 3, part 1 of Theorem 4 shows soundness of non-termination, and parts 2 and 3 - soundness and necessity of computation of natural semantics, respectively.

Unlike reachability, non-termination requires different fixpoint solutions for $N T$ and $\rho$, and thus the order of computation can influence the result. For example, consider checking $E G\left(p c \neq \mathbf{e x}_{1}\right)$ on $\mathrm{EX}_{1}$. Initially, lines 7, 8, and 9 are associated with all the valuations on $x$ and $y$, i.e., $N T(7)=N T(8)=N T(9)=\Sigma_{\{x, y\}}$, and $\rho\left(f_{2}\right)$ is empty, which is not the partial semantics of $f_{2}$ restricted to $N T(9)$. If the computation of $N T$ proceeds along the function call $\mathrm{y}:=\mathrm{f} 2(\mathrm{y})$ using the initial value of $\rho\left(f_{2}\right), N T(8)$ is assigned $\emptyset$. Eventually, $N T(7)=N T(8)=N T(9)=\emptyset$, i.e., the algorithm incorrectly concludes that any execution starting at lines 7,8 or 9 terminates.

The correct order for computing the solution is such that the pre-image of a set $Q$ w.r.t. a function call to $f$ has to be delayed until the derivation of $\rho\left(X_{f}\right)$ w.r.t. $Q$ is finished. Nonetheless, since this order is only restricted to func-call edges, the order of the computation elsewhere can be arbitrary. This can be used to avoid deriving "full" natural semantics. Going back to the previous example, one can first compute $N T$ along all edges except for func-call edges, which will assign $N T(9)$ with $x=2 \wedge y \leq 0$, and then compute natural semantics of $f_{2}$ restricted to the post-condition $z \leq 0$. Similarly, although initially $N T(6)$ is assigned $\Sigma_{\{x, y\}}, N T(6)=(x=2 \wedge y \leq 0)$ after the initial computation of $N T$, which means that only partial natural semantics of $f_{2}$ restricted to the post-condition $z=2$ is needed.

In this section, we have presented mixed semantics - a stack-free operational semantics of PL, and showed how it can be used to check reachability and non-termination of programs with a finite data domain. Although the use of such semantics is not new, our formalization provides a basis for a tight integration between abstraction and analysis, which is described in the next section.

\section{Abstract Reachability and Non-Termination Analysis}

Here, we follow the framework of abstract interpretation (AI) [12] to derive an abstract version of the concrete analysis described in Sec. 4. To do so, we require two abstract domains: abstract sets $A_{s}$ whose elements approximate sets in $\mathbf{2}^{\Sigma}$, and abstract relations $A_{r}$ whose elements approximate relations in $2^{\Sigma \times \Sigma}$. These domains must be equipped with abstract version of all of the operations used in equations reach-otf and nt-otf. Finally, the framework of AI ensures that the solution to an abstract equation is an approximation of the solution to the corresponding concrete equation. In what follows, we identify the necessary abstract operations on $A_{s}$ and $A_{r}$, and then show how to adapt predicate abstraction for our algorithm.

Abstract Domains and Operations. The domain of abstract sets $A_{s}$ must be equipped with a set union $\cup$ (used in the reachability computation) and equality (to detect the fixpoint convergence). The domain of abstract relations $A_{r}$ must be equipped with (a) a pre-image operator to convert abstract relations to abstract transformers over $A_{s}$, (b) asynchronous and sequential compositions of abstract relations (used in natural semantics), (c) scope extension (used to instantiate a function call using natural semantics of a function), and (d) equality (to detect the fixpoint convergence). Furthermore, we need an assume operator that maps an abstract set to a corresponding abstract relation; and, to apply the abstraction directly to the source code, a computable version of ab- 
stract base semantics $\llbracket \cdot \rrbracket_{\alpha}$ that maps each atomic statement $S$ to an abstract relation that approximates $\llbracket S \rrbracket$ (the concrete semantics of $S$ ).

Predicate Abstraction. In the rest of this section, we show how the domain of predicate abstraction $[16,5,18]$ can be extended with the necessary abstract operations to yield abstract reachability and non-termination algorithms.

Predicate abstraction provides domains for abstracting elements, sets, and relations of valuations. Let $V$ be a set of variables, and $\mathcal{P}$ be a set of predicates over $V$. The elementary domain of predicate abstraction over $\mathcal{P}$, denoted $\Theta_{\mathcal{P}}$, is the set of all conjunctions of literals over $\mathcal{P}$. For example, if $\mathcal{P}=\{x>0, x<y\}$, then $\neg(x>0)$ and $(x>0) \wedge \neg(x<y)$ are in $\Theta_{\mathcal{P}}$. An element of $\theta \in \Theta$ approximates any valuation $\sigma \in \Sigma_{V}$ that satisfies all literals in $\theta$. For example, $\sigma=\langle x \mapsto 2, y \mapsto 2\rangle$ is approximated by $x>0$, and is also approximated by $(x>0) \wedge \neg(x<y)$ more precisely.

The elementary domain is lifted to sets and relations in an obvious way: sets over $\Theta$ represent concrete sets, and relations over $\Theta$ - concrete relations. This extension can be either over- or under-approximating, i.e., a collection of concrete valuations corresponding to an abstract set either over-approximates or under-approximates a concrete set. The over- and under-approximating interpretations can also be combined into a single exact-approximation using sets and relations over Belnap logic [18].

Abstract versions of set union, set and relation equality, pre-image, and base semantics for over-approximating predicate abstraction have been defined (e.g., [5]). For example, if $X$ and $Y$ are two abstract sets, their abstract union is $X \cup_{\alpha} Y \triangleq \lambda z$. $X(z) \vee Y(z)$. In $[18,17]$, we show that these operations also naturally extend to underapproximating and exact predicate abstractions. In the latter case, conjunctions and disjunctions, e.g., $\vee$ in the definition of $\cup_{\alpha}$, are interpreted in Belnap logic. We define the missing abstract relational operations assume ${ }_{\alpha}$, asynchronous $\left(\vee_{\alpha}\right)$, and sequential $\left(\circ_{\alpha}\right)$ compositions similarly, using the corresponding definitions from Sec. 2, e.g., if $r_{1}$ and $r_{2}$ are abstract relations, then their abstract asynchronous composition is $r_{1} \vee_{\alpha} r_{2} \triangleq \lambda s, t \cdot r_{1}(s, t) \vee r_{2}(s, t)$, where $\vee$ is interpreted in Boolean logic for overand under-approximating abstraction, and in Belnap logic for exact abstraction.

In concrete semantics, scope extension is used to extend a relation to additional variables. That is, if $r$ is a relation of type $U \rightarrow V$, then $(U \rightarrow U) r$ is an extension of $r$ to variables in $U \backslash V$. In the abstract semantics, relations are defined over predicates; thus, abstract scope extension must extend a relation to additional predicates. To do this, we assume that the elementary abstract domain $\Theta$ corresponding to $U$ can be decomposed into two independent abstract domains: one for $V$ and the other - for $U \backslash V$, i.e., $\Theta$ is defined using predicates that either range only over $V$, or only over $U \backslash V$. Then, abstract scope extension $(\cdot \rightarrow \cdot)_{\alpha}$, defined as in Table 1 , is a sound approximation of concrete scope extension.

Theorem 5. Abstract operations assume $e_{\alpha}, \vee_{\alpha}, \circ_{\alpha}$, and $(\cdot \rightarrow \cdot)_{\alpha}$ as defined above are sound approximations of their concrete counterparts.

In the context of our on-the-fly algorithms, the assumption on abstract scope extension means that predicates that are used to abstract valuations at a return location $l$ of a function call $\mathbf{x}:=f(\mathbf{a})$ are either defined only over $\mathbf{x}$, or only over other variables in the scope of $l$. For example, predicates $x=2$ and $y \leq 0$ can be used to abstract valuations at line 6 in the program $\mathrm{EX}_{1}$, but predicate $x>y$ cannot. This is not a severe restriction 


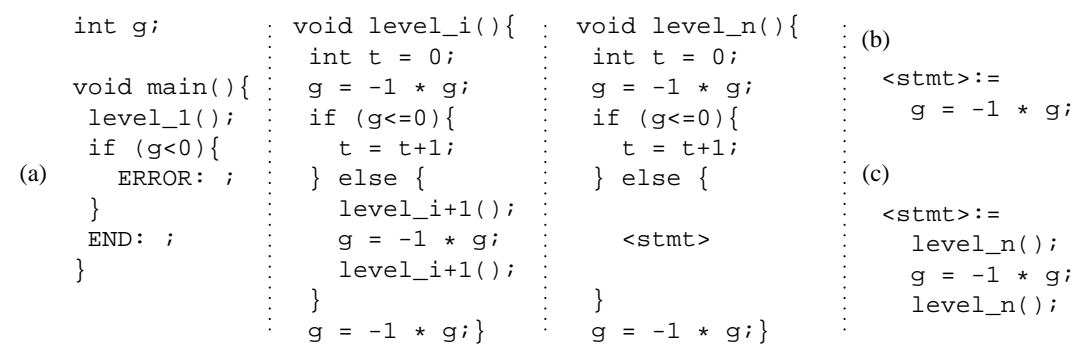

Fig. 3. (a) The template for experiments. (b) <stmt $>$ for template $\mathbf{T} 1(n)$. (c) $<$ stmt $>$ for $\mathbf{T} 2(n)$.

\begin{tabular}{|c||c||c|c|}
\hline \multicolumn{1}{|c||}{} & T1 $(n)$ & \multicolumn{2}{c|}{ T2 $(n)$} \\
\hline$n$ & $E F(p c=$ ERROR $)($ reach $)$ & $E G(p c \neq$ END) (non-terminate) & $\neg E F(p c=$ ERROR) (unreach) \\
\hline \hline 20 & 6.5 & 4.9 & 4.3 \\
\hline 50 & 11.7 & 8.9 & 6.3 \\
\hline 100 & 20.3 & 20.3 & 11.1 \\
\hline 200 & 36.7 & 25.2 & 27.6 \\
\hline 300 & 47.6 & 34.4 & 42.1 \\
\hline 400 & 68.1 & 43.2 & 64.5 \\
\hline 500 & 105.2 & 60.6 & 86.6 \\
\hline
\end{tabular}

Table 3. Experimental results: overall analysis time in seconds.

in practice since a function can always be extended to accept additional parameters and return them without modification.

To summarize, both over- and under-approximating predicate abstractions can be used to soundly abstract reachability and non-termination analysis. The choice depends on the desired algorithm. For example, over-approximation is necessary to establish unreachability, whereas under-approximation - to establish non-termination. Since exact predicate abstraction combines them, it can be used for both verification and refutation.

\section{Experiments}

The technique described in this paper has been implemented in our symbolic software model checker YASM [19]. YASM is written in JAVA; it uses CVC Lite [7] to approximate program statements and CUDD [27] as a decision diagram engine. We have also extended our proof-based refinement approach [17] to handle natural semantics of functions. In the rest of this section, we report on a preliminary evaluation of this implementation. All of the experiments have been conducted on a $2 x \mathrm{P} 4 \mathrm{Xeon}-3.6 \mathrm{GHz}$ server and are available at http://www.cs.toronto.edu/fm/yasm/yasm-tests.zip. Our experiments demonstrate YASM's ability to analyze reachability and non-termination of recursive programs using exact-approximation. In summary:

1. We run YASM on template programs similar to those in the BEBOP and MOPED benchmarks. The experiment shows that the analysis time for both reachability and non-termination increases linearly w.r.t. the number of functions in a program.

2. We show that abstract analysis based on exact-approximation supports both verification and refutation.

3. We compare YASM with MOPED and VERA (BEBOP does not do non-termination), and show that YASM can prove non-termination of the original buggy Quicksort algorithm, whereas MOPED and VERA cannot.

To evaluate the reachability algorithm, we have used the template program $\mathbf{T 1}(n)$ which is a variant of the one used for BEBOP in [6]. T1 $(n)$ is the result of replacing <stmt $>$ in the template shown in Fig. 3(a) with the statements in Fig. 3(b). It consists 
how to compute only the necessary part of natural semantics during the analysis, leading to on-the-fly algorithms for analysis of reachability and non-termination of programs with finite data domains. We then use the framework of abstract interpretation [12] to combine our algorithms with data abstractions, making them applicable to programs with infinite data domains as well. Although we specialize our approach to predicate abstraction, we believe that it can be extended to other abstract domains as well. We have implemented a combination of this approach with exact predicate abstraction in YASM [19] which supports both verification and refutation of properties. Our experiments indicate that YASM scales to programs with a large number of functions and is able to establish non-termination of non-trivial (although small) examples. In particular, we were able to automatically prove non-termination of Ack [10] and Quicksort [14] without any restrictions on the data domain.

In the terminology of interprocedural program analysis [24], our approach is functional since it uses natural semantics to handle function calls. Most other model-checking approaches for recursive programs (e.g., $[23,6,1])$ are functional as well, and only compute the necessary part of natural semantics. Our reachability algorithm can be seen as a pre-image-based variant of the RHS algorithm [23], as implemented in BEBOP [6].

Both MOPED [14] and VERA [1] can check non-termination of programs with finite data domains. Their algorithms are comparable with our non-termination algorithm. However, it is unclear how to combine their techniques with an arbitrary abstraction, whereas it is quite natural in our approach. Note that an ability to detect non-termination of over-approximating Boolean programs is of limited utility since over-approximation often introduces spurious non-terminating computations. Thus, non-termination of an over-approximation says nothing about non-termination of the concrete program.

Jeannet and Serwe [20] apply abstract interpretation to derive abstract analysis of recursive programs by different abstractions of the call stack. Their approach is also parameterized by an arbitrary data abstraction. However, the authors restrict their attention to reachability (i.e., invariance) properties, and do not report on an implementation.

Our interest in non-termination is motivated by the work on termination (e.g., [10]). We view our approach as complementary to that. As illustrated by our experiments, YASM can prove non-termination of non-trivial programs. However, its ability to prove termination is limited to cases where termination can be established by a constant ranking function. In the future, we plan to investigate how the strengths of the two approaches can be combined in a single algorithm.

In this paper, we have restricted our attention to stack-independent properties. We hope to extend our approach to a more general class of properties, e.g., the ones expressible in CARET [2]. Finally, the refinement strategies that are currently implemented in YASM were originally developed for reachability analysis only. While they were sufficient for our non-termination experiments, we believe that strategies specifically tailored to the non-termination analysis are essential for scaling the tool to large programs.

\section{References}

1. R. Alur, S. Chaudhuri, K. Etessami, and P. Madhusudan. "On-the-Fly Reachability and Cycle Detection for Recursive State Machines”. In Proceedings of TACAS'05, volume 3440 of $L N C S$, pages 61-76, 2005.

2. R. Alur, K. Etessami, and P. Madhusudan. "A Temporal Logic of Nested Calls and Returns". In Proceedings of TACAS'04, volume 2988 of LNCS, pages 467-481, 2004. 
3. T. Ball. "Formalizing Counterexample-driven Refinement with Weakest Preconditions". Tech. Report 134, MSR, 2004.

4. T. Ball, O. Kupferman, and G. Yorsh. "Abstraction for Falsification”. In Proceedings of CAV'05, volume 3376 of $L N C S$, pages 67-81, 2005.

5. T. Ball, A. Podelski, and S. Rajamani. "Boolean and Cartesian Abstraction for Model Checking C Programs". STTT, 5(1):49-58, November 2003.

6. T. Ball and S. Rajamani. "Bebop: A Symbolic Model Checker for Boolean Programs". In Proceedings of SPIN'00, volume 1885 of LNCS, pages 113-130, 2000.

7. C. Barrett and S. Berezin. "CVC Lite: A New Implementation of the Cooperating Validity Checker”. In Proceedings of CAV'04, volume 3114 of LNCS, pages 515-518, July 2004.

8. A. Bouajjani, J. Esparza, and O. Maler. "Reachability Analysis of Pushdown Automata: Application to Model-Checking”. In Proceedings of CONCUR'97, volume 1243 of LNCS, pages 135-150, July 1997.

9. G. Bruns and P. Godefroid. "Model Checking Partial State Spaces with 3-Valued Temporal Logics". In Proceedings of CAV'99, volume 1633 of LNCS, pages 274-287. Springer, 1999.

10. B. Cook, A. Podelski, and A. Rybalchenko. "Termination Proofs for System Code". In Proceedings of PLDI'06, pages 415-426, 2006.

11. P. Cousot. "Asynchronous Iterative Methods for Solving a Fixed Point System of Monotone Equations in a Complete Lattice". Research report, Univ. of Grenoble, September 1977.

12. P. Cousot and R. Cousot. "Abstract Interpretation Frameworks". J. of Logic and Computation, 2(4):511-547, 1992.

13. D. Dams, R. Gerth, and O. Grumberg. "Abstract Interpretation of Reactive Systems". ACM Transactions on Programming Languages and Systems, 2(19):253-291, 1997.

14. J. Esparza and S. Schwoon. "A BDD-Based Model Checker for Recursive Programs". In Proceedings of CAV'01, volume 2102 of LNCS, pages 324-336, 2001.

15. P. Godefroid, M. Huth, and R. Jagadeesan. "Abstraction-based Model Checking using Modal Transition Systems”. In CONCUR'01, volume 2154 of LNCS, pages 426-440, 2001.

16. S. Graf and H. Saïdi. "Construction of Abstract State Graphs with PVS". In Proceedings of CAV'97, volume 1254 of $L N C S$, pages 72-83, 1997.

17. A. Gurfinkel and M. Chechik. "Why Waste a Perfectly Good Abstraction?". In Proceedings of TACAS'06, volume 3920 of LNCS, pages 212-226, April 2006.

18. A. Gurfinkel, O. Wei, and M. Chechik. "Systematic Construction of Abstractions for ModelChecking”. In Proceedings of VMCAI'06, volume 3855 of LNCS, pages 381-397, 2006.

19. A. Gurfinkel, O. Wei, and M. Chechik. "YASM: A Software Model-Checker for Verification and Refutation". In Proceedings of CAV'06, volume 4144 of LNCS, pages 170-174, 2006.

20. B. Jeannet and W. Serwe. "Abstracting Call-Stacks for Interprocedural Verification of Imperative Programs". In Proceedings of AMAST'04, volume 3116 of LNCS, 2004.

21. K. Larsen and B. Thomsen. "A Modal Process Logic". In Proceedings of LICS'88, pages 203-210. IEEE Computer Society Press, 1988.

22. H. Nielson and F. Nielson. Semantics with Applications: A Formal Introduction. Wiley Professional Computing, 1992.

23. T. W. Reps, S. Horwitz, and M. Sagiv. "Precise Interprocedural Dataflow Analysis via Graph Reachability”. In Proceedings of POPL'95, pages 49-61, 1995.

24. M. Sharir and A. Pnueli. Program Flow Analysis: Theory and Applications, chapter "Two Approaches to Interprocedural Data Flow Analysis", pages 189-233. Prentice-Hall, 1981.

25. S. Shoham and O. Grumberg. "A Game-Based Framework for CTL Counter-Examples and 3-Valued Abstraction-Refinement”. In Proceedings of CAV'03, volume 2725 of LNCS, pages 275-287. Springer, July 2003.

26. S. Shoham and O. Grumberg. "Monotonic Abstraction-Refinement for CTL". In Proceedings of TACAS'04, volume 2988 of LNCS, pages 546-560, April 2004.

27. F. Somenzi. "CUDD: CU Decision Diagram Package Release”, 2001. 